\title{
Elucidating Functions of FleQ in Xanthomonas oryzae pv. oryzae by Comparative Proteomic and Phenotypic Analyses
}

\author{
Nahee Bae ${ }^{1,+}{ }^{,}$Hye-Jee Park ${ }^{1,+}{ }^{,}$Hanbi Park ${ }^{1}$, Minyoung Kim ${ }^{1}$, Eunsoo Do ${ }^{2}$ \\ and Sang-Wook Han $1, *$ (D) \\ 1 Department of Integrative Plant Science, Chung-Ang University, Anseong 17456, Korea; \\ hi_nahee@daum.net (N.B.); hjks42@hanmail.net (H-J.P.); feelings57@naver.com (H.P.); \\ minyoung0622@naver.com (M.K.) \\ 2 Department of Systems Biotechnology, Chung-Ang University, Anseong 17456, Korea; powerjin19@cau.ac.kr \\ * Correspondence: swhan@cau.ac.kr; Tel.: +82-31-670-3150 \\ + These authors contributed equally to this work.
}

Received: 1 September 2018; Accepted: 2 October 2018; Published: 5 October 2018

\begin{abstract}
To acclimate to different environments, gene expression has to be controlled using diverse transcriptional activators. FleQ activates $\sigma^{54}$-dependent transcription initiation and regulates flagellar biosynthesis and other mechanisms in several bacteria. Xanthomonas oryzae pv. oryzae (Xoo), which is a causal agent of bacterial leaf blight on rice, lacking FleQ loses swimming motility and virulence is not altered. However, other biological mechanisms related with FleQ in Xoo are unknown. In this study, we generated the FleQ-overexpressing strain, Xoo(FleQ), and knockout mutant, Xoo $\Delta f l e Q$. To predict the mechanisms affected by FleQ, label-free shotgun comparative proteomics was carried out. Based on proteomic results, we performed diverse phenotypic assays. $\mathrm{Xoo}(\mathrm{FleQ})$ had reduced ability to elicit disease symptoms and exopolysaccharide production. Additionally, the ability of $X o o \Delta f l e Q(\mathrm{EV})$ (empty vector) and $X o o(\mathrm{FleQ})$ to form biofilm was decreased. Swarming motility of Xoo $\Delta f l e Q(\mathrm{EV})$ was abolished, but was only reduced for $X o o(F l e Q)$. Additionally, abnormal twitching motility was observed in both strains. Siderophore production of $\mathrm{Xoo}(\mathrm{FleQ})$ was enhanced in iron-rich conditions. The proteomic and phenotypic analyses revealed that FleQ is involved in flagellar-dependent motility and other mechanisms, including symptom development, twitching motility, exopolysaccharide production, biofilm formation, and siderophore production. Thus, this study provides fundamental information about a $\sigma^{54}$-dependent transcription activator in Xoo.
\end{abstract}

Keywords: comparative proteomics; Xanthomonas; FleQ; $\sigma^{54}$-dependent transcription activator

\section{Introduction}

Bacterial leaf blight disease caused by Xanthomonas oryzae pv. oryzae (Xoo) is one of the most destructive plant diseases and leads to rice yield losses up to $50 \%$ in countries where rice has been cultivated as a staple crop [1]. Xoo enters leaves through wounds or hydathodes, and spreads in the xylem [2]. White or gray wilt symptoms appear on diseased plants after infection. Xoo produces diverse virulence factors such as exopolysaccharide (EPS), biofilm, extracellular enzymes, and type III effectors [3-5]. Xoo is regarded as an important model bacterium to investigate the molecular mechanisms of plant-bacteria pathogenesis.

To adapt to environments and cause disease, bacteria regulate their gene expression. RNA polymerases interact with sigma $(\sigma)$ factors to initiate transcription, and the sigma factors cooperate with transcription activators [6]. These transcription activators cooperating with a sigma 
factor control gene expression to regulate biological behaviors. For example, PhoB, which is a transcriptional activator with a $\sigma^{70}$ factor, controls regulatory networks under phosphate starvation in Escherichia coli and WhiB7, which activates transcription by interacting with sigma factor SigA, is important for resistance to several antibiotics $[7,8]$. FleQ is known as a $\sigma^{54}$-dependent transcription activator in Pseudomonas aeruginosa, X. campestris pv. campestris (Xcc), and Xoo [9-11].

The participation of FleQ as $\sigma_{-}{ }^{54}$ dependent transcriptional activator has been well-studied in Pseudomonas spp. In P. aeruginosa, FleQ is involved in flagellar-dependent motility, mucin adhesion, and expression of biofilm-related genes $[12,13]$. Transcriptome analysis in P. putida showed that FleQ was related to not only flagellar assembly, but also the type VI secretion system, cyclic diguanylate (c-di-GMP) biosynthesis, and cellulose biosynthesis [14]. In addition, RNA arbitrarily primed polymerase chain reaction revealed that FleQ is involved in regulating the expression of genes encoding topoisomerase, halovibrin A, phosphoglycerate kinase, and potassium efflux proteins in Vibrio fischeri [15]. In Xoo, a fleQ-knockout mutant displayed abolished swimming motility but virulence was not altered on susceptible rice plants [10]. However, other cellular and biological mechanisms controlled by FleQ in Xoo remain unknown.

In this study, we investigated the functions of FleQ (Locus Tag: PXO_00993; Accession Number: ACD59169) by creating a fleQ-knockout mutant, Xoo $\Delta f l e Q$, and a FleQ-overexpressing strain, Xoo(FleQ). A label-free comparative shotgun proteomic analysis and clusters of orthologous groups (COGs) were carried out to assess the biological mechanisms associated with FleQ. Based on proteomic results, diverse phenotypic assays were carried out. The participation of FleQ in Xoo virulence and two types of motility were identified, together with its role in extracellular polysaccharide (EPS) production, biofilm formation, and siderophore production.

\section{Results}

\subsection{FleQ Is Involved in Controlling Expression of Genes Encoding Diverse Proteins}

Although FleQ has been identified as the transcription activator of flagellar synthesis in Xoo [10], other functions related to FleQ are poorly understood and a proteomic analysis for FleQ has not been carried out. To predict biological and cellular mechanisms associated with FleQ, label-free shotgun comparative proteomic analyses were carried out from two sets of strains: $\mathrm{Xoo}(\mathrm{EV})$ (empty vector) vs. Xoo(FleQ) and Xoo vs. Xoo $\Delta f l e Q$. Numbers of detected proteins and peptide spectra matches (PSMs) in biological replicates from LC-MS/MS analysis were shown in Tables S1 and S2. The number of shared proteins in three replicates detected by LC-MS/MS were 1291 and 1244 proteins in Xoo(EV) and $\mathrm{Xoo}(\mathrm{FleQ})$, respectively, and the abundance of these proteins was compared. A total of 174 and 105 proteins were more abundant (>2-fold) in Xoo(EV) and Xoo(FleQ), respectively (Tables S3 and S4). Using COG analysis, these abundant proteins were categorized. Among the 20 groups, the number of proteins in 15 groups were higher in $\mathrm{Xoo}(\mathrm{FleQ})$ than $\mathrm{Xoo}(\mathrm{FleQ})$ (Figure 1A). Proteins belonging group M (cell wall/membrane/envelope biogenesis) are more abundant than other groups, except group $\mathrm{S}$ (function unknown). Interestingly, eight type III effectors (T3Es) (XopF1, XopW, XopN, Hpa3, XopQ, pthoXo1, XopK, XopL), which are modulators of host defense mechanisms, and eight Gum proteins (GumB, GumD, GumH, GumI, GumJ, GumL,GumM, GumN) related to EPS production were more abundant in $\mathrm{Xoo}(\mathrm{EV})$ compared with $\mathrm{Xoo}(\mathrm{FleQ})$ (Table S3). In addition, ferric uptake regulator (Fur) was detected in $\mathrm{Xoo}(\mathrm{FleQ})$, but not in $\mathrm{Xoo}(\mathrm{EV})$, postulating that iron uptake may be affected by overexpression of FleQ. The FleQ protein in Xoo(FleQ) is more abundant (55.73-fold), indicating Xoo(FleQ) is overexpressing FleQ proteins compared with $\mathrm{Xoo}(\mathrm{EV})$.

Between $X o o$ and $X o o \Delta f l e Q, 1227$ and 1161 proteins were shared in the three replicates of Xoo and $X o o \Delta f l e Q$, respectively (Table S2), and the abundance of these proteins was compared. A total of 190 and 56 proteins were more abundant in Xoo and Xoo $\Delta f l e Q$, respectively (Tables S5 and S6). Proteins in 19 groups were predominant in Xoo, excluding W group (Figure 1B) and the number of proteins of $X o o$ in these categories is up to 4 -fold higher than that of $X o o \Delta f l e Q$. Although each group possessed 
fewer than six proteins in $X o o \Delta f l e Q$, group M (cell wall/membrane/envelope biogenesis) and group $P$ (inorganic ion transport and metabolism) contained more than six proteins (Figure 1B). In addition, group K (transcription) was not present in Xoo $\Delta f l e Q$. In contrast to the set, Xoo(EV) vs. Xoo(FleQ), the abundance of only two T3Es, AvrBs2 and Hpa1, was changed in Xoo vs. Xoo $\Delta f l e Q$ (Table S5 and S6). More importantly, group N (cell motility), including a flagellar protein, was not detected in $X o o \Delta f l e Q$, while it was observed in Xoo (Figure 1B).

A

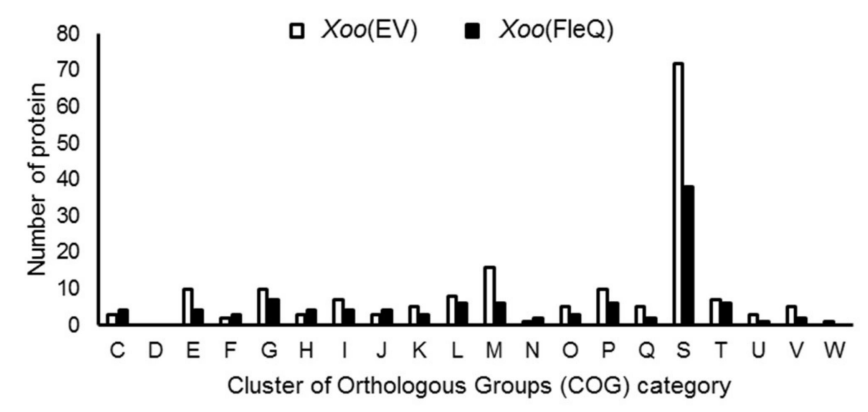

B

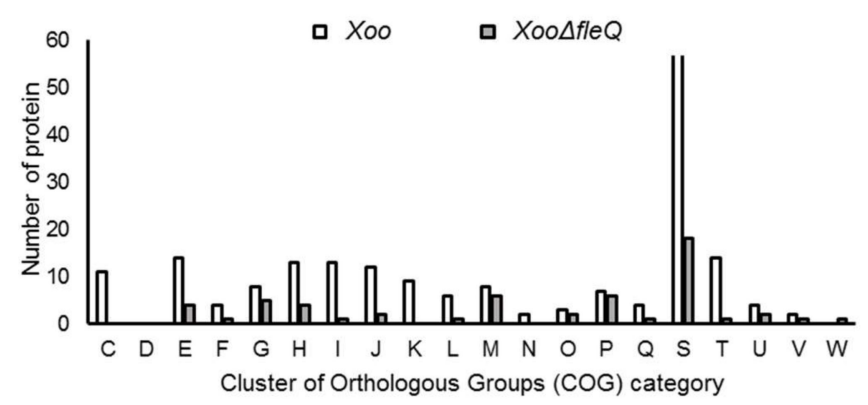

Figure 1. Comparison of the abundance of types of proteins controlled by FleQ in cluster of orthologous groups (COG) groups between (A) Xanthomonas oryzae pv. oryzae, empty vector (Xoo(EV)) vs. Xoo(FleQ) and (B) Xoo vs. Xoo $f f l e Q$. Abbreviations: C, Energy production and conversion; D, Cell cycle control and mitosis; E, Amino acid metabolism and transport; F, Nucleotide metabolism and transport; G, Carbohydrate metabolism and transport; H, Coenzyme metabolism; I, Lipid metabolism; J, Translation; $\mathrm{K}$, Transcription; L, Replication and repair; M, Cell wall/membrane/envelop biogenesis; N, Cell motility; $\mathrm{O}$, Post-translational modification, protein turnover, chaperone functions; $\mathrm{P}$, Inorganic ion transport and metabolism; Q, Secondary structure; R, General functional prediction only; S, Function unknown; $\mathrm{T}$, Signal transduction; U, Intracellular trafficking and secretion; V, Defense mechanisms.

\subsection{Overexpression of FleQ Reduced Symptom Development in Rice Plants}

Virulence in the fleQ-knockout mutant is not altered in Xoo [10]. Therefore, we tested whether the overexpression of the fleQ gene was involved in the virulence of Xoo. We inoculated four strains, $X o o(\mathrm{EV}), X o o(\mathrm{FleQ}), X o o \Delta f l e Q(\mathrm{EV})$, and $\mathrm{Xoo} \Delta f l e Q(\mathrm{FleQ})$, into susceptible hosts (Figure 2A) and measured lesion length at 3, 6, 9, 12, and 15 days after inoculation (DAI) (Figure 2B). Interestingly, $\mathrm{Xoo}(\mathrm{FleQ})$ displayed a reduced lesion length during the entire observation period, while $\mathrm{Xoo} \Delta \mathrm{fleQ}(\mathrm{EV})$ did not. Xoo $\Delta f l e Q(\mathrm{FleQ})$ displayed slightly decreased lesion length in rice plants compared to Xoo(EV) due to copy number of the vector used for complementation. The bacterial population of the four strains was not significantly different in all sections at 15 DAI (Figure 2C). Additionally, there was no significant difference in the growth of the four strains in peptone sucrose broth (PSB) (Figure S1), indicating that FleQ is not involved in bacterial multiplication in the given condition. These results 
indicated that overexpression of FleQ decreased symptom development, although FleQ did not affect Xoo migration and multiplication in rice.

A

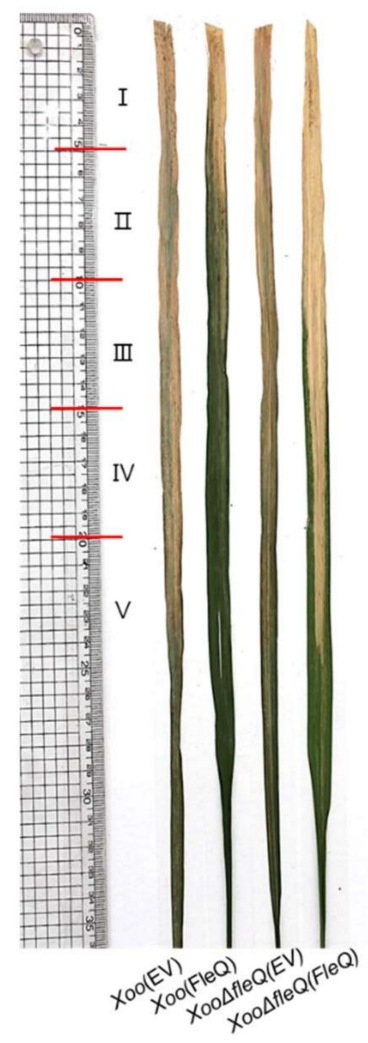

B

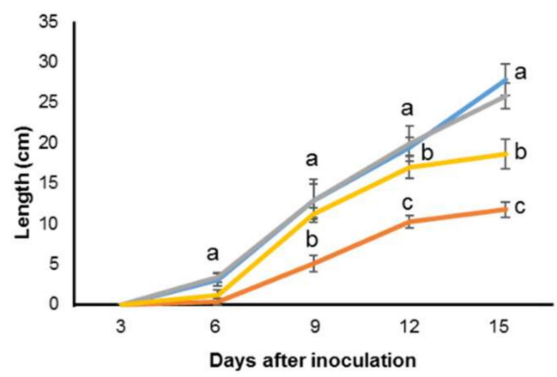

C

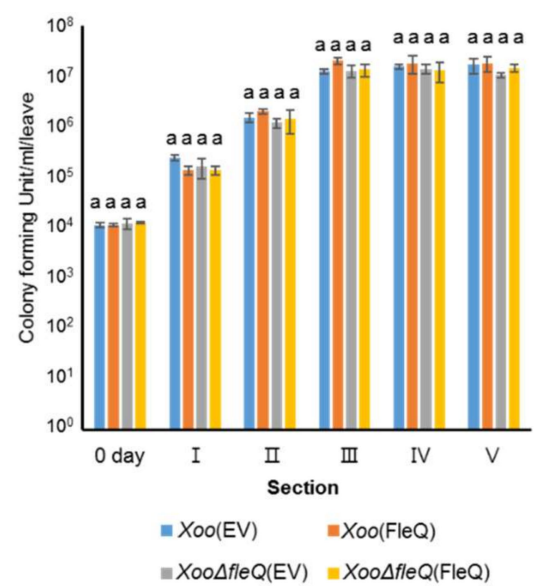

Figure 2. Virulence assay of $X o o(\mathrm{EV}), X o o(\mathrm{FleQ}), X o o \Delta f l e Q(\mathrm{EV})$ and $X o \circ \Delta f l e Q(\mathrm{FleQ})$ strains on rice plants. (A) Representative photograph of leaves taken at 15 days after inoculation (DAI). (B) Graph represents lesion length which was measured at 3, 6, 9, 12, and 15 DAI. (C) Bacterial populations were calculated at day 0 from whole leaves and 15 DAI from sections. Diseased leaves were divided into $5 \mathrm{~cm}$ sections up to $20 \mathrm{~cm}$, and above $20 \mathrm{~cm}$ to the end. Bars indicate the mean of at least six leaves with the standard deviations. Different letters on the bars indicate statistically significant differences in average values by one-way ANOVA $(p<0.05)$.

\subsection{FleQ Affects EPS Production and Biofilm Formation}

Proteomic results indicated Gum proteins related with EPS biosynthesis were more prevalent in $\mathrm{Xoo}(\mathrm{EV})$ than $\mathrm{Xoo}(\mathrm{FleQ})$. Thus, EPS production was tested. EPS production in Xoo(FleQ) was six-fold lower than that in $X o o(\mathrm{EV})$. In the case of $X o o \Delta f l e Q(\mathrm{EV})$, no remarkable difference was shown compared with $\mathrm{Xoo}(\mathrm{EV})$ (Figure 3A). A previous study reported that EPS production is closely associated with biofilm formation and FleQ in P. aeruginosa is involved in biofilm formation [13]. Thus, we investigated biofilm formation (Figure 3B,C). Biofilm formation by Xoo(FleQ) was 1.6-fold decreased compared with $X o o(\mathrm{EV})$, and $\mathrm{Xoo} \Delta f l e Q(\mathrm{EV})$ displayed the least biofilm formation ability among the four strains. The biofilm formation ability of the complemented strain was partially restored. The results suggested that FleQ is associated with EPS production and biofilm formation in Xoo. 
A
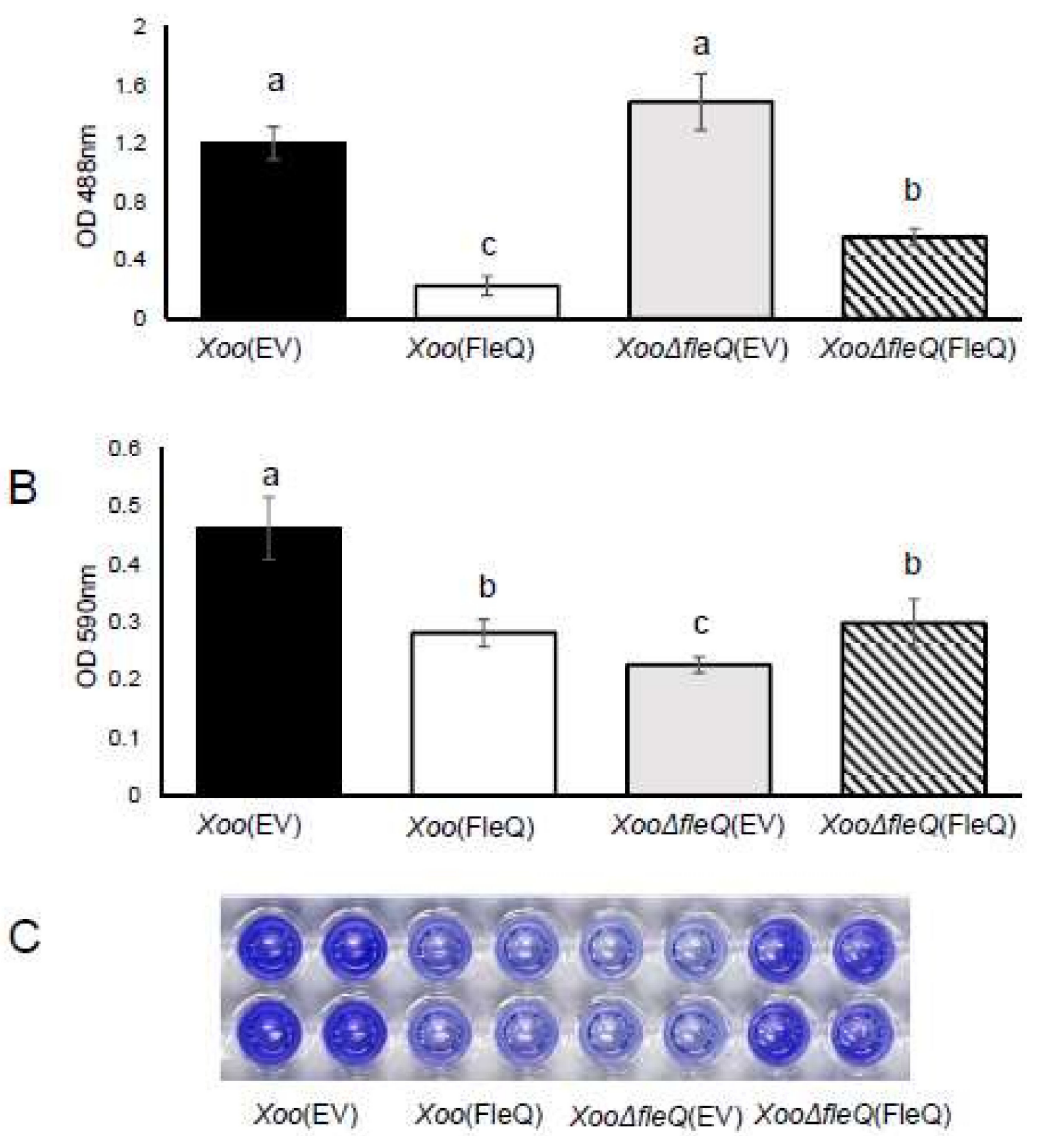

Figure 3. Extracellular polysaccharide (EPS) production and biofilm formation in Xoo strains. (A) After Xoo strains were cultured in peptone sucrose broth for 1 day and in XOM2 for 4 days, EPS was quantified by a phenol-sulfuric acid method at $488 \mathrm{~nm}$. Bars represent the mean of five biological replicates with the standard deviation. (B) Biofilm formation from Xoo strains was determined by the PVC plate assay. Bars indicate the means of 12 biological replicates with the standard deviation. Different letters on the bars indicate statistically significant differences in average values by one-way ANOVA $(p<0.05)$. (C) The photograph of stained cells resolved in 95\% EtOH solution was taken at 7 days after incubation in XOM2. OD = optical density.

\subsection{FleQ Is Crucial for Twitching and Swarming Motility}

A previous study described the loss of swimming ability by a fleQ-knockout mutant in Xoo [10]. Furthermore, group $\mathrm{N}$ (cell motility) was not detected in $X o o \Delta f l e Q(\mathrm{EV})$ in the comparative proteomic results (Figure 1B). To verify if FleQ is required for Xoo motility, swarming and twitching motility were examined. Concerning twitching motility, $\mathrm{Xoo}(\mathrm{EV})$ showed slender and vertical lines and appeared to have a flat edge. By contrast, $X o o(\mathrm{FleQ})$ and $X o o \Delta f l e Q(\mathrm{FleQ})$ had no visible lines and displayed an uneven edge (Figure $4 \mathrm{~A})$. $X o o \Delta f l e Q(\mathrm{EV})$ had thicker, imbalanced lines and a rough edge (Figure $4 \mathrm{~A}$ ). As expected, the swarming motility ability of $\mathrm{Xoo} \Delta f l e Q(\mathrm{EV})$ was abolished and $\mathrm{Xoo}(\mathrm{FleQ})$ also exhibited reduced ability compared with Xoo(EV) (Figure 4B). The motility of the complemented strain was partially restored towards the level of the wildtype. These results clearly indicated that FleQ is involved in flagellar-dependent and flagellar-independent motility in Xoo. 
A

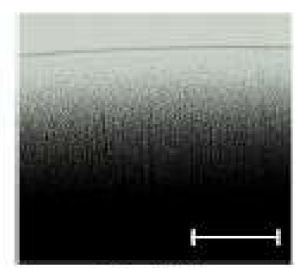

Xoo(EV)

B

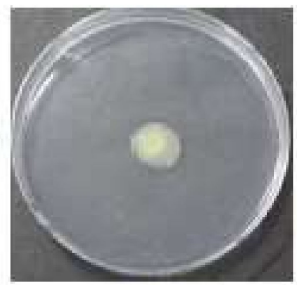

Xoo(EV)

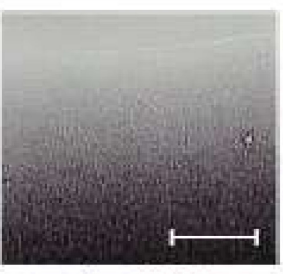

Xoo(FleQ)

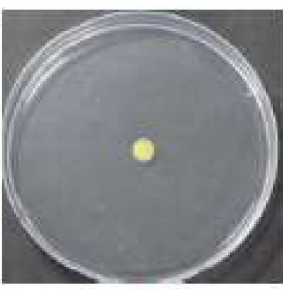

Xoo(FleQ)

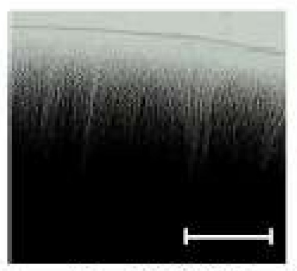

Xoo $\Delta f l e Q(E V)$

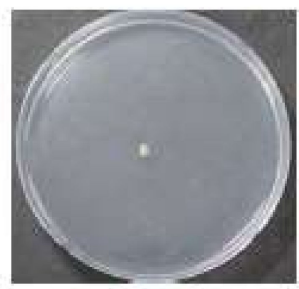

Xoo $\triangle f l e Q(E V)$

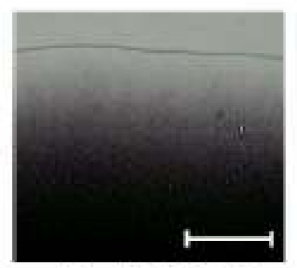

Xoo $\Delta f$ leQ(FleQ)

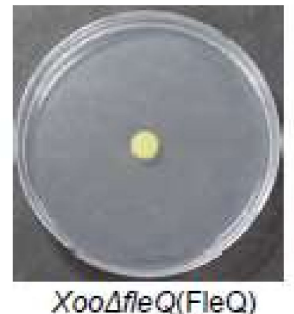

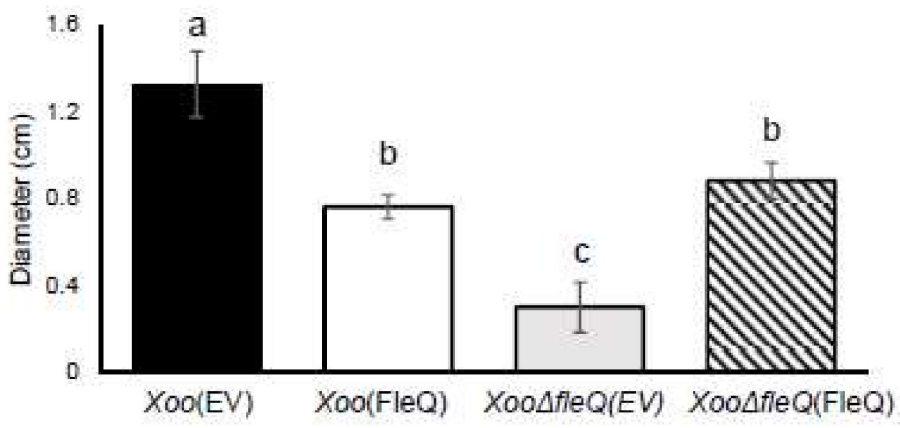

Figure 4. Twitching and swarming motility in Xoo strains. (A) After a 3-day incubation on a peptone sucrose agar (PSA) plate, twitching motility from the colonies was oberved by light microscopy. White bars represent $100 \mu \mathrm{m}$. (B) Swarming motility was observed by a light microscope and analyzed by measuring the diameter of colonies after 7 days incubation on semi-solid XOM2 media. Different letters on the bars indicate statistically significant differences in average values by one-way ANOVA $(p<0.05)$.

\subsection{FleQ Is Involved in Siderophore Production}

Interestingly, the proteomic analysis revealed alterations in the abundance of 18 TonB-dependent receptors, which are membrane-bound and related to the uptake of large substrates like iron-siderophore complexes. Therefore, we investigated the production of the iron-chelating siderophore using the chromeazurol S (CAS) assay. In iron-rich conditions, Xoo(FleQ) produced a 28-fold higher level of siderophores, represented by yellow halos, than Xoo(EV) (Figure 5A). This increase was not observed for $\mathrm{Xoo} \Delta f \mathrm{fle} Q(\mathrm{EV})$. However, when the $\mathrm{Fe}^{2+}$ chelator 2,2'-dipyridyl was added, all strains showed similar siderophore production (Figure 5B). These data indicated that overexpression of FleQ is involved in the facilitation of siderophore production in an iron-rich environment. 


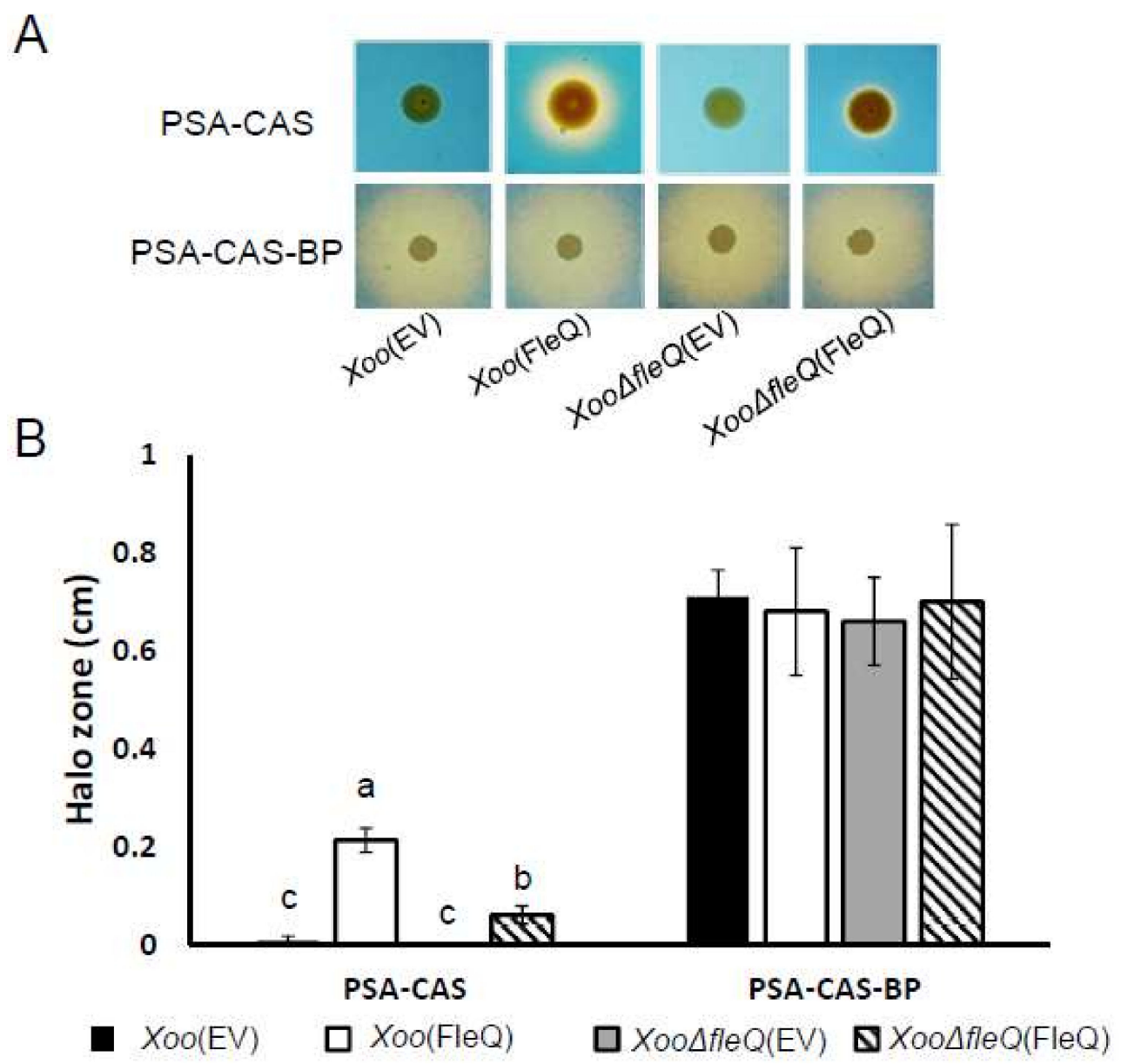

Figure 5. Chromeazurol S (CAS) assay in four strains, Xoo(EV), Xoo(FleQ), Xoo $\Delta f l e Q(\mathrm{EV})$ and $X o o \Delta f l e Q(F l e Q)$. (A) To show colonies on peptone sucrose agar-chromeazurol S (PSA-CAS) and PSA-CAS-BP, which contains 2,2'-dipyridyl as an iron chelator, images were taken after 7 days of incubation. (B) Diameter of comparative halo zones were measuered and means of halo width in each of the four strains was represented by bar graph. Different letters on the bars indicate statistically significant differences in average values by one-way ANOVA $(p<0.05)$.

\section{Discussion}

FleQ is an important factor for flagellar biosynthesis in several bacteria, including Xoo. Although FleQ can affect the expression of flagellar genes [10], there is no evidence of a relationship between FleQ and other mechanisms in Xoo. In this study, we demonstrated functions of FleQ in other biological mechanisms by comparative proteomics and phenotypic observation. In our proteomic analysis, the abundance of eight T3Es and three T3SS proteins was affected by FleQ. Among the T3Es and T3SS, XopQ, XopN, and HrpD6 are important for symptom development in host plants in Xoo [16-18]. A decrease in the level of protein abundance for T3Es and T3SS may cause reduced symptoms on rice leaves in Xoo(FleQ). Our proteomic analysis also showed that eight Gum proteins, including GumD and GumG, were only found in Xoo(EV), and were not present in Xoo(FleQ) (Tables S3 and S4). These proteins are crucial for EPS biosynthesis, which contributes to virulence [19]. Consequently, the ability to produce EPS in Xoo(FleQ) was lower than in Xoo(EV). The reduced EPS production in $\mathrm{Xoo}(\mathrm{FleQ})$ may decrease symptom development. Moreover, Xoo mainly colonizes in the xylem, and blocking xylem cells by EPS produced from Xoo is one of main factors for symptom development [2]. Because Xoo(FleQ) displayed reduced expression of Gum proteins and decreased EPS production, $\mathrm{Xoo}(\mathrm{FleQ})$ may be unable to obstruct water transfer and the development of disease in rice. 
EPS production and biofilm formation is affected by FleQ with c-di GMP in P. aeruginosa [20] and EPS is one of major constituents of biofilms in most bacteria [21]. In agreement with the observations, we demonstrated that the abilities of EPS production and biofilm formation in Xoo(FleQ) were reduced compared with $X o o(\mathrm{EV})$. However, biofilm formation in $X o o \Delta f l e Q(\mathrm{EV})$ was not correlated with EPS production. In several vascular pathogens, including Pantoea stewartii, Ralstonia solanacearum, and Dickeya dadantii, flagella are involved in biofilm formation [22-24]. In addition, flagella are also important for mature biofilm formation in X. axonopodis pv. citri (Xac) [25]. Xac lacking flagella contain fewer water channels, which enable nutrient flow to bacterial cells in the biofilm architecture, and thin biofilm structures were observed. Our proteomic analysis revealed that Xoo(EV) only showed flagellar proteins compared with $X o o \Delta f l e Q(E V)$ (Tables S3 and S4) and that swarming motility was abolished in the $X o o \Delta f l e Q(E V)$ mutant. Therefore, it can be postulated that low biofilm formation of $X o o \Delta f l e Q(E V)$ is caused by lack of flagellum in the mutant.

Twitching motility is a flagellar-independent motility essential for host colonization and formation of biofilms in some bacteria [26]. Type IV pili (T4P), which mediate twitching motility and pili retraction, are thought to be the motive force for twitching motility [27]. Although flagellar proteins were not detected in Xoo $\Delta f l e Q$ (Table S5), a PilW protein, one of the T4P proteins, was found in $X o o \Delta f l e Q$ in our proteomic results (Table S6). The T4P protein, FimC, was found on Xoo(EV), but not in Xoo(FleQ) (Tables S3 and S4). Additionally, abnormal twitching motility was observed in $\mathrm{Xoo}(\mathrm{FleQ})$ and $\mathrm{Xoo} \Delta f l e Q(\mathrm{EV})$ (Figure $4 \mathrm{~A}$ ), which is consistent with proteomic results. FleQ is important for Xoo swimming motility, which is influenced by rotation of unipolar flagella, and Xcc flagellar synthesis $[10,11]$. In an agreement with these studies, we also showed that swarming motility of $\mathrm{Xoo} \Delta f l e Q(\mathrm{EV})$ was abolished. Thus, proteomic and phenotypic analyses indicate that FleQ is involved in flagellar-dependent and independent motility.

Iron is an essential element for survival and bacteria employ siderophores to efficiently uptake $\mathrm{Fe}^{3+}$ from outside sources via transport involving TonB-dependent receptors [28]. In Xoo, the strain lacking RpfF displayed overproduction of siderophores and were less virulent than the wildtype [29]. Similarly, Xoo(FleQ) increased siderophore production in an iron-rich environment and had reduced ability to show disease symptoms. Additionally, proteomic results reveal that FleQ could be associated with regulating expression of some TonB-dependent proteins. Thus, it can be speculated that the increased siderophore production reduced disease symptoms. These results support the proposition that iron metabolism contributes to the virulence of Xanthomonas spp.

In conclusion, we have postulated biological mechanisms related to FleQ by a label-free shotgun, comparative proteomic analysis in Xoo. We further showed that FleQ is associated with symptom development, EPS production, biofilm formation, motility, and siderophore production by phenotypic observation. Thus, FleQ is involved in the regulation of not only flagellar biosynthesis, but also other biological mechanisms in Xoo. This study will provide new insight into understanding the functions of FleQ.

\section{Materials and Methods}

\subsection{Bacterial Strains and Growth Condition}

For this study, X. oryzae pv. oryzae (Xoo) strain PXO99A was used as the wildtype; its genome was completely sequenced [30]. Xoo strains were cultured in peptone sucrose medium (peptone: $10 \mathrm{~g} / \mathrm{L}$, sucrose: $10 \mathrm{~g} / \mathrm{L}$, L-glutamic acids: $1 \mathrm{~g} / \mathrm{L})$ and XOM2 medium $(670 \mu \mathrm{M}$ DL-methionine, $10 \mathrm{mM}$ sodium L-glutamate, $14.7 \mathrm{mM} \mathrm{KH}_{2} \mathrm{PO}_{4}, 40 \mu \mathrm{M} \mathrm{MnSO}_{4}, 240 \mu \mathrm{M} \mathrm{Fe(III)-EDTA,} \mathrm{0.18 \%} \mathrm{D-xylose,} \mathrm{and} 5 \mathrm{mM}$ $\mathrm{MgCl}_{2}, \mathrm{pH}$ 6.5) at $28{ }^{\circ} \mathrm{C}$. Escherichia coli strain DH5 $\alpha$ was grown in Lysogeny broth medium [31]. Four antibiotics were used: gentamycin $(10 \mu \mathrm{g} / \mathrm{mL})$, kanamycin $(50 \mu \mathrm{g} / \mathrm{mL})$, cephalexin $(30 \mu \mathrm{g} / \mathrm{mL})$, and ampicillin $(100 \mu \mathrm{g} / \mathrm{mL})$. 


\subsection{Generation of Xoo Strains}

All plasmids and bacterial strains used in this study are listed in Table S7. To generate the insertional mutant, $X o o \Delta f l e Q$, the DNA fragment containing fle $Q$ was amplified by gene specific primers ( $5^{\prime}$-atgatgccgagcatcgtgaa- $3^{\prime}$ and $5^{\prime}$-gatttcgcctcgaccatcct- $\left.5^{\prime}\right)$. The amplified product was cloned into the pGEM-T-easy vector and a kanamycin cassette from pUC4k was inserted in the middle of the fleQ gene digested by KpnI. The construct was introduced into Xoo by electroporation. $X o o \Delta f l e Q$ was selected on PSA medium containing kanamycin, but not ampicillin. To create the $f l e Q$-overexpressing strain, $X o o(F l e Q)$, the open reading frame of $f l e Q$ was amplified with the gene specific primers (5'-ctcgagatgagtgagtcccgcattctgt- $3^{\prime}$ and $5^{\prime}$-aagcttcagtggtggtggtggtggtggttggccagctcggtctgctcg- $\left.3^{\prime}\right)$. The amplified DNA was cloned into a pGEM-T-easy vector. The identified DNA fragment was subcloned again into a pBBR1MCS-5 vector [32] and the expression of FleQ was driven by the Lac promoter in the vector, creating pBBR1FleQ. The construct was introduced into $X o o$ and $X o o \Delta f l e Q$ to generate $X o o(F l e Q)$ and the complemented strain, $X o o \Delta f l e Q(F l e Q)$, respectively. The pBBR1MCS-5 was transferred into Xoo and $\mathrm{Xoo} \Delta f l e Q$, creating $\mathrm{Xoo}(\mathrm{EV})$ and $\mathrm{Xoo} \Delta f l e Q(\mathrm{EV})$.

\subsection{Label-Free Shotgun Proteomic Analysis}

Incubation and harvest of $\mathrm{Xoo}(\mathrm{FleQ})$ and $\mathrm{Xoo} \Delta f l e Q$ strains, protein extraction, peptide preparation, liquid chromatography-tandem mass spectrometry (LC-MS/MS), peptide identification, quantification, and statistical analysis were performed as described previously [33]. Briefly, Xoo strains were incubated in PSB, washed, adjusted to an $\mathrm{OD}_{600}$ of 0.6 in XOM2 medium, and then grown again for $16 \mathrm{~h}$. After harvesting bacterial cells, total proteins were extracted and trypsin was used for the digestion. Tryptic-digested peptides $(2 \mu \mathrm{g})$ of three biological replicates were analyzed using split-free nano-liquid chromatography (LC, EASY-nLC II; Thermo Fisher Scientific, Waltham, MA, USA) linked to the LTQ Velos Pro instrument (Thermo Fisher Scientific) equipped with Thermo Proteome Discoverer 1.3 software (ver. 1.3.0.399). A column packed in-house with $7.5 \mathrm{~cm}$ of MAGIG C18AQ 200A $(5 \mu \mathrm{m})$ material (Michrom BioResources, Auburn, CA, USA) was used for separation. Six data-dependent MS/MS scans were used to acquire full mass spectrometry spectra. Dynamic exclusion was allowed with one repeat count, $0.5 \mathrm{~min}$ repeat duration, and exclusion duration of $3 \mathrm{~min}$, with charge state selection permitted to take $2^{+}$and $3^{+}$ions by priority. The six most intense ions in each full MS scan were assembled for fragmentation and explored in centroid mode within the linear ion trap. SEQUEST software was used to analyze the obtained MS/MS spectra. The Xoo strain PXO99A database was used to search spectra. With opposite database searches, all collected peptides had a false discovery rate (FDR) of 0.01 and precursor mass exactness of $100 \mathrm{ppm}$. The probability score for all peptides was $>20$. The least two unique peptides were matched with proteins which were regarded as present in the sample. The obtained data were imported into Scaffold 4 (Proteome Software, Portland, OR, USA). Identified proteins and PSMs for Xoo(EV) and Xoo were used in a study published recently [34] and in this study for the comparison. Peptide spectra matches (PSMs) of each protein were normalized from total PSMs. The average number of normalized PSMs in three biological replicates was calculated per protein and used as a comparison value to identify differently (over two-fold) abundant proteins in two sets, $X o o$ vs. $X o o \Delta f l e Q$ and $X o o(E V)$ vs. Xoo(FleQ). To postulate the mechanisms, clusters of orthologous groups (COG) classification were employed.

\subsection{Virulence Test}

Oryza sativa L. (cultivar kitaake (Kit)) plants [35] were grown in a greenhouse at $25-32{ }^{\circ} \mathrm{C}$ for 5 weeks. Xoo strains were grown in PSA and adjusted to an $\mathrm{OD}_{600}$ of 0.6. A leaf clipping method was performed. After inoculation, lesion length was checked on the infected leaves at 3, 6, 9, 12, and 15 days after inoculation. Infected leaves were divided into five groups: I (0-5), II (6-10), III (11-15), IV (16-20), and V (>21 cm). Each section was sliced into small pieces and immersed in $10 \mathrm{mM} \mathrm{MgCl}_{2}$. The extracted bacterial suspensions were serially diluted and dropped onto PSA 
containing appropriate antibiotics to enumerate the bacterial population. The bacterial population was assessed by colony counting. The assay was repeated at least four times with six biological replicates.

\subsection{Motility Assay}

Twitching motility was performed as described previously [36]. Briefly, Xoo strains grown for 2 days were adjusted to an $\mathrm{OD}_{600}$ of 0.6 and tested on PSA media. After $3 \mu \mathrm{L}$ bacterial resuspension was dotted onto plates, bacteria were incubated for 3 days and observed using a light microscope (Olympus SZX10, Tokyo, Japan). This experiment was performed at least four times with four biological replicates. Swarming motility was conducted as previously described [37]. Swarming motility was investigated on media containing $0.3 \%$ agar. Three microliters of bacterial suspension $\left(\mathrm{OD}_{600}\right.$ of 1.0$)$ were dropped onto the motility plates which were incubated at $28^{\circ} \mathrm{C}$ for 7 days. The diameter of colony expansion was measured. The assay was repeated at least three times with five biological replicates.

\subsection{EPS Production and Biofilm Formation}

EPS production was determined as described previously [38]. To obtain maximum EPS, Xoo strains were adjusted to an $\mathrm{OD}_{600}$ of 0.3 , serially diluted (1000-fold), and grown in $5 \mathrm{~mL}$ PSB for 5 days. One milliliter of bacterial cultures was centrifuged for $3 \mathrm{~min}$ at $10,500 \times \mathrm{g}$ and $400 \mu \mathrm{L}$ of the supernatant was mixed in $1.2 \mathrm{~mL}$ of $95 \%$ ethanol. After storing at $-20^{\circ} \mathrm{C}$ for 1 day, the stored samples were centrifuged for $10 \mathrm{~min}$ at $16,500 \times \mathrm{g}$ at $4{ }^{\circ} \mathrm{C}$, desiccated for 1 hour, and dissolved in $1 \mathrm{~mL}$ of ultrapure water. One hundred microliters of the samples were added to $900 \mu \mathrm{L}$ water, $1 \mathrm{~mL}$ of $5 \%$ aqueous phenol and $5 \mathrm{~mL}$ of $\mathrm{H}_{2} \mathrm{SO}_{4}$ in cold conditions. Mixed samples were measured at $488 \mathrm{~nm}$ by a spectrophotometer. Mean values from five biological replicates were determined and this experiment was repeated at least four times. Biofilm formation was measured by the polyvinyl chloride (PVC) microplate method as reported previously [37]. Xoo cells were incubated in PSB and adjusted to an $\mathrm{OD}_{600}$ of 0.3 . One hundred microliters of bacterial suspensions were resuspended in $10 \mathrm{~mL}$ of XOM2 and incubated in PVC plates containing XOM2 media at $28{ }^{\circ} \mathrm{C}$ for 5 days. After eliminating supernatants, the remaining cells were stained using $0.1 \%$ crystal violet, washed two times with water, and dissolved in $95 \%$ ethanol. The dissolved samples were measured at $590 \mathrm{~nm}$ by a Spectramax 190 microplate reader (Molecular devices, Sunnyvale, CA, USA). This experiment was repeated six times with twelve biological replicates.

\subsection{Siderophore Production}

The chromeazurol S (CAS) assay was used to examine siderophore production as described previously [38]. PSA-CAS plates were used as the iron-rich medium. For the limited iron condition, 2,2'-dipyridyl was added in the PSA-CAS plates (PSA-CAS-BP). Xoo strains were incubated in PSA, harvested, washed, and adjusted to an $\mathrm{OD}_{600}$ of 0.6. Three microliters of bacterial suspension were dropped onto the plates. The halo zones were measured on plates after incubation for 7 days at $28^{\circ} \mathrm{C}$. This experiment was repeated six times with four biological replicates.

\subsection{Statistical Analyses}

All statistical analyses were accomplished using SPSS 12.0K (Statistical Package for the Social Sciences; SPSS Inc., Chicago, IL, USA). ANOVA and Tukey's multiple comparison were used to determine significant difference $(p<0.05)$.

Supplementary Materials: Supplementary materials can be found at http:/ /www.mdpi.com/1422-0067/19/10/ 3038/s1.

Author Contributions: S.W.H. conceived the study. N.B., H.J.P., H.P., and M.K. conducted all the experiments. N.B., H.J.P., H.P., M.K., E.D., and S.W.H. analyzed the data. N.B., H.J.P., and S.W.H. wrote the main manuscript text. All authors reviewed the manuscript. 
Funding: This work was supported by the Next-Generation BioGreen 21 Program [PJ01328901] of the Rural Development and by Basic Science Research Program through the National Research Foundation of Korea funded by the Ministry of Education [NRF-2018R1D1A1B07045724], Republic of Korea (to S.W. Han).

Conflicts of Interest: The authors declare no conflict of interest.

\section{References}

1. Reddy, V.S.; Kumar, Y.N.; Raghavendra, A.; Sowjenya, G.; Kumar, S.; Ramyasree, G.; Reddy, G.R.; Kangueane, P. In silico model of DSF synthase RpfF protein from Xanthomonas oryzae pv. oryzae: A novel target for bacterial blight of rice disease. Bioinformation 2012, 8, 504-507. [PubMed]

2. Nino-Liu, D.O.; Ronald, P.C.; Bogdanove, A.J. Xanthomonas oryzae pathovars: Model pathogens of a model crop. Mol. Plant Pathol. 2006, 7, 303-324. [CrossRef] [PubMed]

3. Das, A.; Rangaraj, N.; Sonti, R.V. Multiple adhesin-like functions of Xanthomonas oryzae pv. oryzae are involved in promoting leaf attachment, entry, and virulence on rice. Mol. Plant Microbe Interact. 2009, 22, 73-85. [PubMed]

4. Singh, V.B.; Kumar, A.; Isaac Kirubakaran, S.; Ayyadurai, N.; Sunish Kumar, R.; Sakthivel, N. Comparison of exopolysaccharides produced by Xanthomonas oryzae pv. oryzae strains, BXO1 and BXO8 that show varying degrees of virulence in rice (Oryza sativa L.). J. Phytopathol. 2006, 154, 410-413.

5. Sinha, D.; Gupta, M.K.; Patel, H.K.; Ranjan, A.; Sonti, R.V. Cell wall degrading enzyme induced rice innate immune responses are suppressed by the type 3 secretion system effectors XopN, XopQ, XopX and XopZ of Xanthomonas oryzae pv. oryzae. PLoS ONE 2013, 8, e75867. [CrossRef] [PubMed]

6. Studholme, D.J.; Dixon, R. Domain architectures of sigma54-dependent transcriptional activators. J. Bacteriol. 2003, 185, 1757-1767. [CrossRef] [PubMed]

7. Blanco, A.G.; Canals, A.; Bernues, J.; Sola, M.; Coll, M. The structure of a transcription activation subcomplex reveals how sigma(70) is recruited to PhoB promoters. EMBO J. 2011, 30, 3776-3785. [CrossRef] [PubMed]

8. Burian, J.; Yim, G.; Hsing, M.; Axerio-Cilies, P.; Cherkasov, A.; Spiegelman, G.B.; Thompson, C.J. The mycobacterial antibiotic resistance determinant WhiB7 acts as a transcriptional activator by binding the primary sigma factor SigA (RpoV). Nucleic Acids Res. 2013, 41, 10062-10076. [CrossRef] [PubMed]

9. Jyot, J.; Dasgupta, N.; Ramphal, R. FleQ, the major flagellar gene regulator in Pseudomonas aeruginosa, binds to enhancer sites located either upstream or atypically downstream of the RpoN binding site. J. Bacteriol. 2002, 184, 5251-5260. [CrossRef] [PubMed]

10. Tian, F.; Yu, C.; Li, H.; Wu, X.; Li, B.; Chen, H.; Wu, M.; He, C. Alternative sigma factor RpoN2 is required for flagellar motility and full virulence of Xanthomonas oryzae pv. oryzae. Microbiol. Res. 2015, 170, 177-183. [CrossRef] [PubMed]

11. Hu, R.M.; Yang, T.C.; Yang, S.H.; Tseng, Y.H. Deduction of upstream sequences of Xanthomonas campestris flagellar genes responding to transcription activation by FleQ. Biochem. Biophys. Res. Commun. 2005, 335, 1035-1043. [CrossRef] [PubMed]

12. Arora, S.K.; Ritchings, B.W.; Almira, E.C.; Lory, S.; Ramphal, R. A transcriptional activator, FleQ, regulates mucin adhesion and flagellar gene expression in Pseudomonas aeruginosa in a cascade manner. J. Bacteriol. 1997, 179, 5574-5581. [CrossRef] [PubMed]

13. Hickman, J.W.; Harwood, C.S. Identification of FleQ from Pseudomonas aeruginosa as a c-di-GMP-responsive transcription factor. Mol. Microbiol. 2008, 69, 376-389. [CrossRef] [PubMed]

14. Wang, Y.; Li, Y.; Wang, J.; Wang, X. FleQ regulates both the type VI secretion system and flagella in Pseudomonas putida. Biotechnol. Appl. Biochem. 2017, 65, 419-427. [CrossRef] [PubMed]

15. Millikan, D.S.; Ruby, E.G. FlrA, a sigma54-dependent transcriptional activator in Vibrio fischeri, is required for motility and symbiotic light-organ colonization. J. Bacteriol. 2003, 185, 3547-3557. [CrossRef] [PubMed]

16. Gupta, M.K.; Nathawat, R.; Sinha, D.; Haque, A.S.; Sankaranarayanan, R.; Sonti, R.V. Mutations in the predicted active site of Xanthomonas oryzae pv. oryzae XopQ differentially affect virulence, suppression of host innate immunity, and induction of the HR in a nonhost plant. Mol. Plant. Microbe Interact. 2015, 28, $195-206$.

17. Cheong, H.; Kim, C.Y.; Jeon, J.S.; Lee, B.M.; Sun Moon, J.; Hwang, I. Xanthomonas oryzae pv. oryzae type III effector XopN targets OsVOZ2 and a putative thiamine synthase as a virulence factor in rice. PLoS ONE 2013, 8, e73346. 
18. Guo, X.; Zou, H.; Li, Y.; Zou, L.; Chen, G. HrpD6 gene determines Xanthomonas oryzae pv. oryzae to trigger hypersensitive response in tobacco and pathogenicity in rice. Wei Sheng Wu Xue Bao 2010, 50, 1155-1163. [PubMed]

19. Wang, J.C.; So, B.H.; Kim, J.H.; Park, Y.J.; Lee, B.M.; Kang, H.W. Genome-wide identification of pathogenicity genes in Xanthomonas oryzae pv. oryzae by transposon mutagenesis. Plant Pathol. 2008, 57, 1136-1145. [CrossRef]

20. Maunders, E.; Welch, M. Matrix exopolysaccharides; the sticky side of biofilm formation. FEMS Microbiol. Lett. 2017, 364, fnx120. [CrossRef] [PubMed]

21. Danese, P.N.; Pratt, L.A.; Kolter, R. Exopolysaccharide production is required for development of Escherichia coli K-12 biofilm architecture. J. Bacteriol. 2000, 182, 3593-3596. [CrossRef] [PubMed]

22. Tans-Kersten, J.; Huang, H.; Allen, C. Ralstonia solanacearum needs motility for invasive virulence on tomato. J. Bacteriol. 2001, 183, 3597-3605. [CrossRef] [PubMed]

23. Jahn, C.E.; Willis, D.K.; Charkowski, A.O. The flagellar sigma factor FliA is required for Dickeya dadantii virulence. Mol. Plant Microbe Interact. 2008, 21, 1431-1442. [CrossRef] [PubMed]

24. Herrera, C.M.; Koutsoudis, M.D.; Wang, X.; von Bodman, S.B. Pantoea stewartii subsp. stewartii exhibits surface motility, which is a critical aspect of Stewart's wilt disease development on maize. Mol. Plant Microbe Interact. 2008, 21, 1359-1370. [CrossRef] [PubMed]

25. Malamud, F.; Torres, P.S.; Roeschlin, R.; Rigano, L.A.; Enrique, R.; Bonomi, H.R.; Castagnaro, A.P.; Marano, M.R.; Vojnov, A.A. The Xanthomonas axonopodis pv. citri flagellum is required for mature biofilm and canker development. Microbiology 2011, 157, 819-829. [PubMed]

26. Mattick, J.S. Type IV pili and twitching motility. Annu. Rev. Microbiol. 2002, 56, 289-314. [CrossRef] [PubMed]

27. Russell, M.A.; Darzins, A. The pilE gene product of Pseudomonas aeruginosa, required for pilus biogenesis, shares amino acid sequence identity with the N-termini of type 4 prepilin proteins. Mol. Microbiol. 1994, 13, 973-985. [CrossRef] [PubMed]

28. Wandersman, C.; Delepelaire, P. Bacterial iron sources: From siderophores to hemophores. Annu. Rev. Microbiol. 2004, 58, 611-647. [CrossRef] [PubMed]

29. Chatterjee, S.; Sonti, R.V. rpfF mutants of Xanthomonas oryzae pv. oryzae are deficient for virulence and growth under low iron conditions. Mol. Plant Microbe Interact. 2002, 15, 463-471. [PubMed]

30. Salzberg, S.L.; Sommer, D.D.; Schatz, M.C.; Phillippy, A.M.; Rabinowicz, P.D.; Tsuge, S.; Furutani, A.; Ochiai, H.; Delcher, A.L.; Kelley, D.; et al. Genome sequence and rapid evolution of the rice pathogen Xanthomonas oryzae pv. oryzae PXO99A. BMC Genomics 2008, 9, 204.

31. Bertani, G. Lysogeny at mid-twentieth century: P1, P2, and other experimental, systems. J. Bacteriol. 2004, 186, 595-600. [CrossRef] [PubMed]

32. Kovach, M.E.; Elzer, P.H.; Hill, D.S.; Robertson, G.T.; Farris, M.A.; Roop, R.M.; Peterson, K.M. Four new derivatives of the broad-host-range cloning vector pBBR1MCS, carrying different antibiotic-resistance cassettes. Gene 1995, 166, 175-176. [CrossRef]

33. Park, H.J.; Bae, N.; Park, H.; Kim, D.W.; Han, S.W. Comparative proteomic analysis of three Xanthomonas spp. cultured in minimal and rich media. Proteomics 2017, 17, 1700142. [CrossRef] [PubMed]

34. Bae, N.; Park, H.J.; Park, H.; Kim, M.; Han, S.W. Deciphering the functions of the outer membrane porin OprBXo involved in virulence, motility, exopolysaccharide production, biofilm formation, and stress tolerance in Xanthomonas oryzae pv. oryzae. Mol. Plant Pathol. 2018, in press. [CrossRef] [PubMed]

35. Kim, S.L.; Choi, M.; Jung, K.H.; An, G. Analysis of the early-flowering mechanisms and generation of T-DNA tagging lines in Kitaake, a model rice cultivar. J. Exp. Bot. 2013, 64, 4169-4182. [CrossRef] [PubMed]

36. Park, H.J.; Park, C.J.; Bae, N.; Han, S.W. Deciphering the role of tyrosine sulfation in Xanthomonas oryzae pv. oryzae using shotgun proteomic analysis. Plant. Pathol. J. 2016, 32, 266-272. [PubMed]

37. Park, H.J.; Jung, H.W.; Han, S.W. Functional and proteomic analyses reveal that $w x c B$ is involved in virulence, motility, detergent tolerance, and biofilm formation in Xanthomonas campestris pv. vesicatoria. Biochem. Biophys. Res. Commun. 2014, 452, 389-394. [CrossRef] [PubMed]

38. Nguyen, M.P.; Park, J.; Cho, M.H.; Lee, S.W. Role of DetR in defence is critical for virulence of Xanthomonas oryzae pv. oryzae. Mol. Plant Pathol. 2016, 17, 601-613. [CrossRef] [PubMed]

(C) 2018 by the authors. Licensee MDPI, Basel, Switzerland. This article is an open access article distributed under the terms and conditions of the Creative Commons Attribution (CC BY) license (http:/ / creativecommons.org/licenses/by/4.0/). 